\title{
Genome-wide identification and expression profiling of invertase gene family for abiotic stresses tolerance in Poncirus trifoliata
}

\author{
Bachar Dahro ${ }^{1,2}$, Yue Wang ${ }^{1}$, Ahmed Alhag ${ }^{1}$, Chunlong Li ${ }^{1}$, Dayong Guo ${ }^{1 *}$ and Ji-Hong Liu*
}

\begin{abstract}
Background: Sucrose (Suc) hydrolysis is directly associated with plants tolerance to multiple abiotic stresses. Invertase (INV) enzymes irreversibly catalyze Suc degradation to produce glucose (Glc) and fructose (Frc). However, genome-wide identification and function of individual members of the INV gene family in Poncirus trifoliata or its Citrus relatives in response to abiotic stresses are not fully understood.

Results: In this report, fourteen non-redundant PtrINV family members were identified in P. trifoliata including seven alkaline/neutral INV genes (PtrA/NINV1-7), two vacuolar INV genes (PtrVINV1-2), and five cell wall INV isoforms (PtrCWINV1-5). A comprehensive analysis based on the biochemical characteristics, the chromosomal location, the exon-intron structures and the evolutionary relationships demonstrated the conservation and the divergence of PtrINVs. In addition, expression analysis of INV genes during several abiotic stresses in various tissues indicated the central role of A/NINV7 among INV family members in response to abiotic stresses. Furthermore, our data demonstrated that high accumulation of Suc, Glc, Frc and total sugar contents were directly correlated with the elevated activities of soluble INV enzymes in the cold-tolerant P. trifoliata, C. ichangensis and C. sinensis, demonstrating the potential role of soluble INV enzymes for the cold tolerance of Citrus.
\end{abstract}

Conclusions: This work offered a framework for understanding the physiological role of INV genes and laid a foundation for future functional studies of these genes in response to abiotic stresses.

Keywords: P. trifoliata, Invertase, Genome-wide identification, Sucrose hydrolysis, Gene expression, Abiotic stresses

\section{Background}

Abiotic stresses including cold, salinity, and drought cause cellular osmotic stress leading to cell membrane damage and protein structure disorder. Additionally, these environmental cues increase the oxidative status of plants by perturbing the delicate equilibrium between the production and the detoxification of reactive oxygen species (ROS) $[1,2]$. As a response to the

\footnotetext{
*Correspondence: guoday@mail.hzau.edu.cn; liujihong@mail.hzau.edu.cn ${ }^{1}$ Key Laboratory of Horticultural Plant Biology (MOE), College of Horticulture and Forestry Science, Huazhong Agricultural University, Wuhan 430070, China

Full list of author information is available at the end of the article
}

stressful conditions, plants induce the accumulation of compatible solutes like sucrose (Suc), fructose (Fru) and glucose (Glc) [3]. The accumulation of osmoprotectants maintains water retention in the plant cells without interrupting normal metabolism in a process called osmotic adjustment [4]. Osmotic adjustment sustains the cellular turgor for plant growth and survival during stressful environments and increases its capacity to detoxify ROS $[3,5]$. Thus, the ability of plants to orchestrate carbon assimilation and sugar metabolism could determine plant survival during stressful environments $[4,6]$.

Primarily, carbohydrates generated by photosynthesis are either transiently stored as starch or reversed to Suc and transported to the heterotrophic organs [7]. 
Under environmental stresses, starch is converted to Suc as energy supply to support plant survival when photosynthesis becomes insufficient [8]. Therefore, Suc is an essential molecule that accumulated after short-term of abiotic stresses and it is positively correlated with abiotic stress tolerance of plants [9-11]. However, Suc cannot be used directly and its catabolism is necessary to be involved in abiotic stresses responsive pathways [12-14].

In plants, Suc can be catalyzed reversibly by the Suc synthase enzyme (SuSy, EC 2.4.1.13) in the presence of UDP to produce UPD-Glc and Frc in the cytoplasm, which provides substrates for starch and proteins biosynthesis [15]. Meanwhile, the invertase enzyme (INV, EC 3.2.1.26) irreversibly hydrolyzes Suc to form two hexoses (Glc and Frc) [16]. It has been reported that INV members can be clustered into acidic INVs (AINV) and structurally unrelated alkaline/neutral INVs (A/NINV) based on the similarity of protein sequence and the biochemical property of their $\mathrm{pH}$ optima $[17,18]$. Furthermore, AINV proteins are disturbed into cell wall invertase (CWINV) and vacuole invertase (VINV) according to the protein subcellular localization [19, 20]; and A/NINV proteins are diverged into several distinct isoforms localized in cell membrane, cytoplasm, nucleus, chloroplast, and mitochondria [13, 20-22]. Unlike CWINVs and VINVs that act at acidic $\mathrm{pH}(4.5-5.5)$, A/NINVs function at optimal pH of 7.0-7.8 [23]. Both VINV and A/NINVs are soluble proteins with an acidic $p I$, while CWINVs are insoluble with basic $p I$ to facilitate the interaction with the cell wall [23].

Physiological and genetic evidences indicated to the conserved functions of CWINV genes in plant growth, pollen fertility, oval formation, seed and fruit development, and pathogens defense through regulating the Suc unloading to sink tissues and controlling the sugar signals $[19,24,25]$. Besides, VINV isoforms were revealed to regulate hexoses accumulation and sucrose metabolism in fruit [26]. On contrary, several reports demonstrated that the $A / N I N V$ s play more prominent roles than the $A I N V$ s during stressful conditions [27]. For example, the Arabidopsis thaliana AtA/NINVG controls osmotic stress-induced developmental process [28]. Moreover, the wheat alkaline $T a-A-I n v$ was revealed to play a role in response to environmental stresses [29]. Arabidopsis mitochondrial AtA/NINVC and AtA/NINVA maintain the function of mitochondria and thus contribute in facilitating the energy demands and the ROS homeostasis for plant stress tolerance [30, 31]. In parallel, Arabidopsis chloroplastic AtA/NINVE regulates carbon balance between the cytosol and the plastids, affecting the function of photosynthetic apparatus [32, 33]. However, INV enzymes activities and gene expression profiles showed diverged manner of response in different plant tissue under various abiotic stresses, indicating that $I N V$ genes function distinguishably and un-redundantly during environmental stresses [27, 30,31]. In consideration of their important roles, INV gene family have been previously reported in Arabidopsis [31, 34], rice [16], tomato [21], pepper [35], cassava [36, 37], sugarcane [27], and maize [38]. However, $I N V$ genes have not been identified and characterized systematically in Citrus, an economically important fruit crop cultivated worldwide.

Most of the Citrus cultivars do not have best combination between fruit qualitative and quantitative traits with environmental stresses tolerance traits. Thus, the genetic improvement for new Citrus varieties is necessary to overcome the barriers of production [39]. The biological nature of Citrus growth makes the conventional breeding programs incompetent to achieve the genetic improvement of Citrus [40-42]. Therefore, modern biotechnologies and genetic engineering are promising approaches to beat the disadvantages of traditional breeding programs [43]. In this regard, identifying genetic mechanisms involved in response to abiotic stresses becomes essential for the genetic improvement programs of Citrus [44].

Despite the advanced progress in the genome sequencing of Citrus species $[43,45,46]$, genome-wide identification of INV gene family has not studied yet in Citrus. In this report, we performed genome-wide characterization and expression profiling analysis of INV family members from the cold-hardy $P$. trifoliata (the common rootstock of Citrus). Furthermore, we assessed the relationship between the accumulation of sugars and the INVs' activity in the Citrus and its relative species. Consequently, our study lays a foundation for functional characterization of $I N V$ genes in abiotic stresses tolerance, especially for cold response in Citrus.

\section{Materials and methods}

\section{Plant materials and growth conditions}

To obtain insight about the freezing tolerance of Citrus species, six Citrus and its relative species (Ptr; P. trifoliata, Ci; C. ichangensis, Cs; C. sinensis, Cg; C. grandis, $\mathrm{Cl}, \mathrm{C}$. limon and Fj; Fortunella japonica) were harvested from the National Center of Citrus Breeding at Huazhong Agricultural University, Wuhan, China. The seedlings were grown in soil and placed in controlled chamber with the following conditions: $14 \mathrm{~h}$ light $/ 10 \mathrm{~h}$ dark cycle, constant temperature of $23^{\circ} \mathrm{C}, 65 \%$ relative humidity, and uniform illumination of $120 \mu \mathrm{mol} . \mathrm{m}^{-2} \cdot \mathrm{s}^{-1}$ (Unless otherwise stated, all plant growth conditions are the same).

For cold treatment, two month-old seedlings growing in soil were exposed to $4{ }^{\circ} \mathrm{C}$ for indicated times $(0 \mathrm{~h}, 6 \mathrm{~h}$, $12 \mathrm{~h}, 24 \mathrm{~h}, 48 \mathrm{~h}, 72 \mathrm{~h}$ ), and then recovered at room temperature for a day. For salinity treatment, two month-old plants growing in soil were transferred to $200 \mathrm{mM} \mathrm{NaCl}$ 
solution for indicated times $(0 \mathrm{~h}, 6 \mathrm{~h}, 12 \mathrm{~h}, 24 \mathrm{~h}, 48 \mathrm{~h}$, $72 \mathrm{~h}$ ), and then recovered at water for a day. For dehydration treatment, two month-old plants were extracted from soil and putted on filter paper at room temperature for indicated times $(0 \mathrm{~h}, 0.5 \mathrm{~h}, 1 \mathrm{~h}, 2 \mathrm{~h}, 3 \mathrm{~h}, 5 \mathrm{~h})$, and then transferred to water for recovery. Plant materials from determined time points were harvested and directly immersed in liquid $\mathrm{N}_{2}$ and preserved at $-80^{\circ} \mathrm{C}$ for further analysis. P. trifoliata flowers of spring blossom and matured $P$. trifoliata fruits were collected from the National Center of Citrus Breeding at Huazhong Agricultural University. All assessments were performed with three biological replicates.

\section{RNA extraction and quantitative real-time PCR (qRT-PCR) analysis}

Total RNA was extracted from plant materials using a Trizol reagent (Aidlab, China) and treated with DNase I (Thermo, USA) to eliminate the DNA contamination. The RevertAid Reverse transcriptase cDNA synthesis Kit (Thermo, USA) was used to synthesize the first strand of cDNA. AceQ qPCR SYBR Green Master Mix (Vazyme, China) was utilized according to the manufacturer's instructions to perform qRT-PCR via Applied Biosystems ${ }^{\circledR}$ QuantStudio $^{\text {TM }} 7$ Flex Real-Time PCR System (ABI, USA). The gene expression levels were determined using gene-specific primers that designed with the aid of the primer designing tool of NCBI (https://www.ncbi.nlm. nih.gov/tools/primer-blast/). While, Actin was used as an internal reference gene to normalize the gene expression (unless otherwise stated, all primers are listed in Supplementary Table 1 ). The specificity of primer pairs were verified by semi-quantitative reverse transcription polymerase chain reaction (RT-PCR) and by melting curves. The cycling program comprised an initial denaturation step at $94^{\circ} \mathrm{C}$ for $5 \mathrm{~min}$, followed by 45 cycles of $94^{\circ} \mathrm{C}$ for $10 \mathrm{~s}$ and $60^{\circ} \mathrm{C}$ for $30 \mathrm{~s}$. The gene expression analysis for each treatment or each time point was performed in four technical replicates from three biological replicates and representative data are shown as the mean values \pm SE. The relative expression profile was calculated using the $2^{-\Delta \Delta C T}$ method relatively to the initial treatment time $(0 \mathrm{~h})$ that set as 1 . The absolute gene expression was applied to analyze the transcription of $I N V$ genes in different tissue types according to previous report [47].

\section{Identification and cloning of INV gene family members}

To identify the $I N V$ family members in P. trifoliata, the sequence of Arabidopsis INV members were used as queries for BLAST searching against $C$. sinensis in phytozome v13 tool (https://phytozome-next.jgi.doe.gov/ blast-search), and C. sinensis and P. trifoliata genomes in sweet orange annotation project datasets (http://citrus. hzau.edu.cn/cgi-bin/orange). The sequences annotated as INV members with zero $E$-value were selected for further analysis. INV genes were cloned from the synthesized cDNA according the manufacturer's instruction of the $2 \mathrm{X}$ Phanta Max Master Mix Kit (Vazyme, China). The purified PCR products were cloned into the $\mathrm{PTOPO}$ vector using pTOPO-Blunt simple cloning kit (Aidlab, China) according to the manufacturer's instruction, and then transformed into Escherichia coli (E. coli) competent cells DH5 $\alpha$. The transformed bacteria were grown on selective solid LB medium containing appropriate antibiotic for overnight. The positive colonies were confirmed by PCR and them sequenced by Wuhan AuGCT DNA-SYN CO.LTD (China). All of used primers were listed in Supplementary Table 1.

\section{Bioinformatics analysis}

The sequences obtained by the cloning were used as queries to search for the full-length open reading frame (ORF) using the ORF finder from NCBI (https://www. ncbi.nlm.nih.gov/orffinder/). In addition, the theoretical isoelectric points $(p I)$ and molecular weights (MW) of INV proteins were calculated using ProtParam tool (https://web.expasy.org/protparam/). Multiple protein sequence alignment was performed using Clustal Omega, and visualized by GeneDoc. Analysis of conserved domains was done by SMART tool (http://smart. embl-heidelberg.de/). To study the phylogenetic relationship, multiple alignment of putative INV proteins from Arabidopsis thaliana, Oryza sativa, Vitis vinifera, Zea may, Malus domestica [21, 48] was applied on MEGAX software to generate the phylogenetic tree using the neighbor-joining algorithm. Bootstraps with 1000 replicates for Poisson correction model were performed to assess node support. The putative subcellular localization of INV proteins was predicted using TargetP, ChloroP, Mitoprot, and Yloc tools [49-53]. The conserved motif of PtrINVs were identified by the Multiple EM for Motif Elicitation (MEME Suite 5.4.1) server under the default setting (https://meme-suite.org/meme/tools/ meme). Gene structures accompanied with phylogenetic tree were built using the Gene Structure Display Server (GSDS) (http://gsds.gao-lab.org/index.php). Chromosomal location of INV genes was designed using TBtool [54]. Cis-acting elements were identified using the PlantCARE database (http://bioinformatics.psb.ugent.be/ webtools/plantcare/html/).

\section{Measurement of INV, SuSy and HXK enzymes' activity}

The activities of INV enzymes were measured according to the previous reports with minor modification $[55,56]$. Frozen samples were ground to a fine powder using a pre-cold mortar and pestle. Total proteins were 
extracted from $0.3 \mathrm{~g}$ of ground samples using $3 \mathrm{~mL}$ of precooled extraction buffer $(40 \mathrm{mM}$ Tris- $\mathrm{HCl} \mathrm{pH} 7.6,1 \mathrm{mM}$ EDTA, $3 \mathrm{mM} \mathrm{MgCl}_{2}, 1 \mathrm{mM}$ benzamidine, $0.1 \mathrm{mM}$ PMSF, $24 \mu \mathrm{M}$ NADP, $14 \mathrm{mM} \beta$-mercaptoethanol) on pre-cooled mortar until material was fully thawed $(20-30 \mathrm{~min})$. The homogenate was collected and centrifuged at 10,000 $\times g$ and $4{ }^{\circ} \mathrm{C}$ for $10 \mathrm{~min}$. Subsequently, the crude extracts of soluble proteins were re-centrifuged as above for $20 \mathrm{~min}$ to discard any contamination. The soluble crude was dialyzed against $20 \mathrm{mM}$ potassium phosphate buffer (PBK) $(\mathrm{pH} 7.4)$ at $4{ }^{\circ} \mathrm{C}$ for $12 \mathrm{~h}$ to remove the soluble sugars. The solid pellet was rinsed three times with $\mathrm{ddH}_{2} \mathrm{O}$ for $10 \mathrm{~min}$ to remove soluble proteins. The washed pellet was re-suspended in $1 \mathrm{ml}$ of the pre-cooled extraction buffer with high salt concentration $(1 \mathrm{M} \mathrm{NaCl})$, and shook gently at $4^{\circ} \mathrm{C}$ for $12 \mathrm{~h}$ in dark. The homogenate was centrifuged twice as above for $10 \mathrm{~min}$ to extract the cell wall bound proteins. The cell wall crude was dialyzed against $20 \mathrm{mM}$ PBK buffer ( $\mathrm{pH} 7.4$ ) at $4{ }^{\circ} \mathrm{C}$ for $12 \mathrm{~h}$ to remove the soluble sugars and $\mathrm{NaCl}$. All dialyzed extracts were divided into small aliquots and were snap-frozen in liquid $\mathrm{N}_{2}$ and preserved at $-20^{\circ} \mathrm{C}$ for further analysis.

The activity of INV enzymes was tested according to $[55,56]$ with slight modification. To evaluate A/ NINV enzyme activity, $20 \mu \mathrm{l}$ of dialyzed extracts of soluble protein was incubated at $37^{\circ} \mathrm{C}$ for $1 \mathrm{~h}$ with $180 \mu \mathrm{l}$ of reaction buffer ( $50 \mathrm{mM}$ Bicine- $\mathrm{KOH}$ buffer, $\mathrm{pH} 7.6$ ) containing $100 \mathrm{mM}$ Suc. To assess VINV and CWINV enzymes activities, $20 \mu \mathrm{l}$ of dialyzed extracts from supernatant or pellet fractions, respectively, were incubated at $37^{\circ} \mathrm{C}$ for $1 \mathrm{~h}$ with $180 \mu \mathrm{l}$ of reaction buffer $(50 \mathrm{mM}$ sodium acetate at $\mathrm{pH} 4.5)$ containing $100 \mathrm{mM}$ Suc. Assays without reaction were utilized as control to remove the background. The INV activities were estimated by evaluating Glc content produced by Suc hydrolysis and presented as $\mu$ mol Glc. mg protein ${ }^{-1}$. min $^{-1}$. Twenty microliter of dialyzed extract from the supernatant was applied to determine hexokinase (HXK) and SuSy activities via suitable commercial kits according to the manufacturer's instructions (Nanjing Jiancheng Bioengineering Institute, Nanjing, China).

\section{Measurement of biochemical and physiological parameters}

Glc content was measured spectrophotometrically by detecting the absorbance change at $505 \mathrm{~nm}$ according to the kit's instruction (Nanjing Jiancheng Bioengineering Institute, Nanjing, China). Suc and Frc levels were assessed by particular kits following the manufacturer's instructions (Nanjing Jiancheng Bioengineering Institute, Nanjing, China). Total sugar contents were examined by an appropriate kit (BC2710, Solarbio, China). Glc6-phosphate (G-6-P) was enzymaticly tested by using a specific kit (ab83426, Abcam, China). EL\%, chlorophyll $a$ fluorescence, $\mathrm{Fv} / \mathrm{Fm}$ ratio, the histochemical staining of nitro blue tetrazolium (NBT) and 3, 3'-diaminobenzidine (DAB) were conducted according to previous reports [13, 57].

\section{Statistical analysis}

Experiments were performed using completely randomized design. Statistical analyses were performed using Statistical Package for the Social Sciences (SPSS) software. The significant difference are analyzed using one-way Analysis of variance (ANOVA) method, and displayed as $\left({ }^{*} P<0.05,{ }^{* * *} P<0.01,{ }^{* * * *} P<0.001\right)$. Error bars refer to $\pm \operatorname{SE}(n=3)$.

\section{Results}

Genome-wide mining identified fourteen PtrINV genes in $P$. trifoliata

To identify PtrINV genes in P. trifoliata genome, Arabidopsis INV genes were used as query to BLAST the reference genomes of several Citrus species deposited in sweet orange annotation project datasets. All of the redundant genes were removed after a similarity sequence comparison. Finally, 14 non-redundant $I N V$ genes were identified. Seven putative A/NINV isoforms (PtrA/NINV1-7), two putative VINV isoforms (PtrVINV1-2) and five putative CWINV isoforms (PtrCWINV1-5) were cloned from the cDNA of $P$. trifoliata (Table 1). According to the sequence analysis, the full-length open reading frame (ORF) of seven PtrA/NINV members ranged from 1677 bp (PtrA/NINV3) to $2037 \mathrm{bp}$ (PtrA/NINV7); with putative protein sequences within the range 558-678 amino acids (aa) and molecular weights (MW) within the range $63.7-76.7 \mathrm{kDa}$ (Table 1). The multiple sequence alignment of $\mathrm{A} / \mathrm{NINV}$ proteins showed that the $\mathrm{C}$-terminal of A/NINV proteins has a highly conserved glycoside hydrolase 100 (glyco-hydro-100) domain (Fig. S1), indicating that the variable $\mathrm{N}$-terminus could contribute in the variation of the subcellular localization [29]. Moreover, the high similarity in the protein sequence between PtrA/NINV1 and PtrA/NINV6, PtrA/NINV2 and PtrA/ NINV7, and PtrA/NINV3 and PtrA/NINV4 indicates that they are should be paralogous pairs. For the PtrAINV genes, the ORF ranged from $1203 \mathrm{bp}$ (PtrCWINV3) to $2070 \mathrm{bp}$ (PtrVINV1), and the length of the putative protein ranged from 573 to 687 aa with MW ranged from 64.4 to $76.4 \mathrm{kDa}$ (Table 1 ). The putative protein sequences of the PtrVINV1, PtrVINV2, PtrCWINV1, PtrCWINV2, PtrCWINV4, and PtrCWINV5 contain typical motifs of plant AINVs, including the Suc-binding motif (NDPNG), the transition-state stabilizer motif (RDP), and the cysteine catalytic motif (MWECV/PD) (Fig. S2) [26]. The sequence comparison of AINV proteins elucidated that 


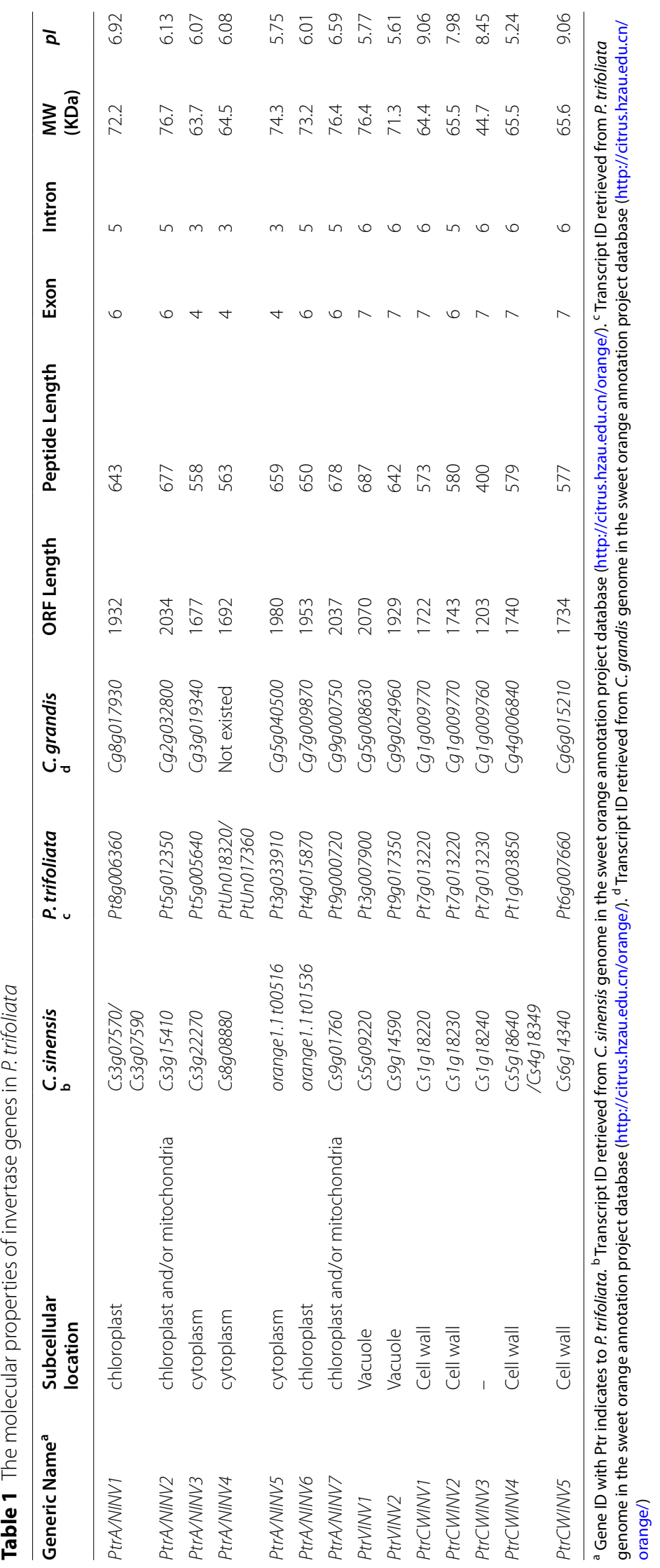


the N-terminal of VINV proteins contains longer signal sequence compared to that of CWINVs (Fig. S2).

As we know, the protein localization is closely related its function. The prediction of subcellular localization of PtrINVs was conducted by using different database and tools, like Mitoprot, TargetP, ChloroP and YLoc tools [49-52]. Accordingly, PtrA/NINV3, PtrA/NINV4 and PtrA/NINV5 were estimated to be localized in the cytoplasm with no transit peptide (Table 1). PtrA/NINV1 and PtrA/NINV6 proteins were predicted to have chloroplast transit signal. Furthermore, PtrA/NINV2 and PtrA/NINV7 proteins were found to have chloroplast and mitochondria transit signals (Table 1) which was consistent with the chloroplast and mitochondria localization of PtrA/NINV7 in the previous work [13]. On the other hand, YLoc tool identified vacuole signal in the PtrVINV1 and PtrVINV2 proteins (Table 1). The dedicated Targetp server recognized secretory signals in the protein sequences of PtrCWINV1, PtrCWINV2, PtrCWINV4 and PtrCWINV5 (Table 1). However, PtrCWINV3 is non-functional protein and the prediction tool does not identify secretory signal in its amino acid sequence. The various locations of INV proteins in different cellular compartments indicate to the diverse functions of these proteins in sucrose catabolism and sugar signaling transduction.

\section{The phylogenetic relationship and gene structure of PtrINV family members}

To classify the phylogenetic relationships of INV proteins, we constructed a phylogenetic tree based on 19, 21, 17, 17, 18 and 14 INV members from Oryza sativa, Zea may (monocots), Arabidopsis thaliana (dicot), Vitis vinifera, Malus domestica and P. trifoliata (fruit trees), respectively. The phylogenetic analyses showed that the INV family can be classified into two distinct groups (AINV and A/NINV enzymes), and each of these two groups can be further divided into multi-subgroups (Figs. 1 and 2). The phylogenetic tree elucidated a faster divergence in the AINV proteins in comparison with the A/NINV paralogs (Figs. 1 and 2). VINV proteins are branched off from CWINV paralogs during the evolution. Similarly, mitochondria and chloroplast localized INVs were divided from cytoplasmic INVs (Figs. 1 and 2). Interestingly, both AINV and A/NINV proteins of monocots, dicots and fruit trees were clustered into distinct clades (Fig. 1), suggesting that INV proteins were evolved after monocots, dicots and fruit trees divergence. Based on sequence alignment, PtrAINV proteins were classified into VINV (PtrVINV1 and PtrVINV2) and CWINV groups (PtrCWINV1-5) (Fig. 1). The PtrCWINV1, PtrCWINV2 and PtrCWINV3 isoforms are direct tandem repeats and clustered into one clade (Fig. 1). On the other hand,
PtrA/NINV3, PtrA/NINV4 and PtrA/NINV5 were clustered into cytoplasm-localized clade (Fig. 1). Furthermore, PtrA/NINV1 and PtrA/NINV6 were diverged into chloroplast-targeted clade, and PtrA/NINV2 and PtrA/ NINV7 were grouped into mitochondria-targeted branch (Fig. 1). Multiple sequence alignments and phylogenetic analysis revealed that INV proteins from Oryza sativa, Zea may (monocots), Arabidopsis thaliana (dicot), Vitis vinifera, Malus domestica and P. trifoliata shared high levels of similarity, which suggests similar functions for those homologous members.

For the gene structure analysis, the cytoplasmic localized $A / N I N V$ genes contain 3 introns and 4 exons, while the mitochondria and chloroplast localized $A / N I N V$ isoforms have 5 introns and 6 exons (Fig. 2B). Besides, all of $A I N V$ genes have 7 exons and 6 introns, except for 6 exons and 5 introns are identified in PtrCWINV2 genomic sequence. Apart from the conserved gene structure, all $A I N V$ genes have the second small $9 \mathrm{bp}$ exon (GATCCT/C/AAAT/C) that encodes DPN amino acid of the Suc-binding motif (NDPNG) (Fig. 2B) [26, 58]. For the protein conserved motif, 3 to 6 typical motifs were identified in INVs protein sequence. In general, members with close phylogenetic relationships had high sequence similarity and similar motifs (Fig. 2C-D). The presence of the same type of conserved motifs might indicate functional similarity among PtrINV family members.

\section{The chromosomal location of PtrINV genes in P. trifoliata}

Chromosomal location analysis showed that 13 PtrINV genes were distributed evenly on eight of the nine chromosomes (Chr) of P. trifoliata (Fig. 3), except for Chr 2. PtrA/NINV4 was located on unattributed scaffold. PtrA/ NINV genes were found on Chr 3, Chr 4, Chr 5, Chr 8 and Chr 9. Besides, our analysis showed that PtrVINV1 and PtrVINV2 are located on Chr 3 and Chr 9, respectively. Moreover, PtrCWINV1, PtrCWINV2 and PtrCWINV3 isoforms are direct tandem repeat on the Chr 7 of $P$. trifoliata. PtrCWINV4 and PtrCWINV5 are located on the Chr 1 and Chr 6, respectively.

Furthermore, we juxtaposed the distributions of $I N V$ genes within the genomes of Citrus species (C. sinensis, $C$. grandis and $P$. trifoliata). Interestingly, the result demonstrated that $A / N I N V 1-3$ genes were located on one chromosome (Chr 3 ) in C. sinensis, but they were found in two chromosomes (Chr 5, Chr 8) in P. trifoliata, and in three chromosomes (Chr 2, Chr 3, Chr 8) in C. grandis (Table 1). Moreover, A/NINV4 was located on Chr 8 of $C$. sinensis and absent in the reference genome of $C$. grandis (Table 1). This diverse chromosome distribution pattern of $I N V$ genes between different Citrus species indicated that genetic variations was happened in the evolutionary process of the Citrus. 


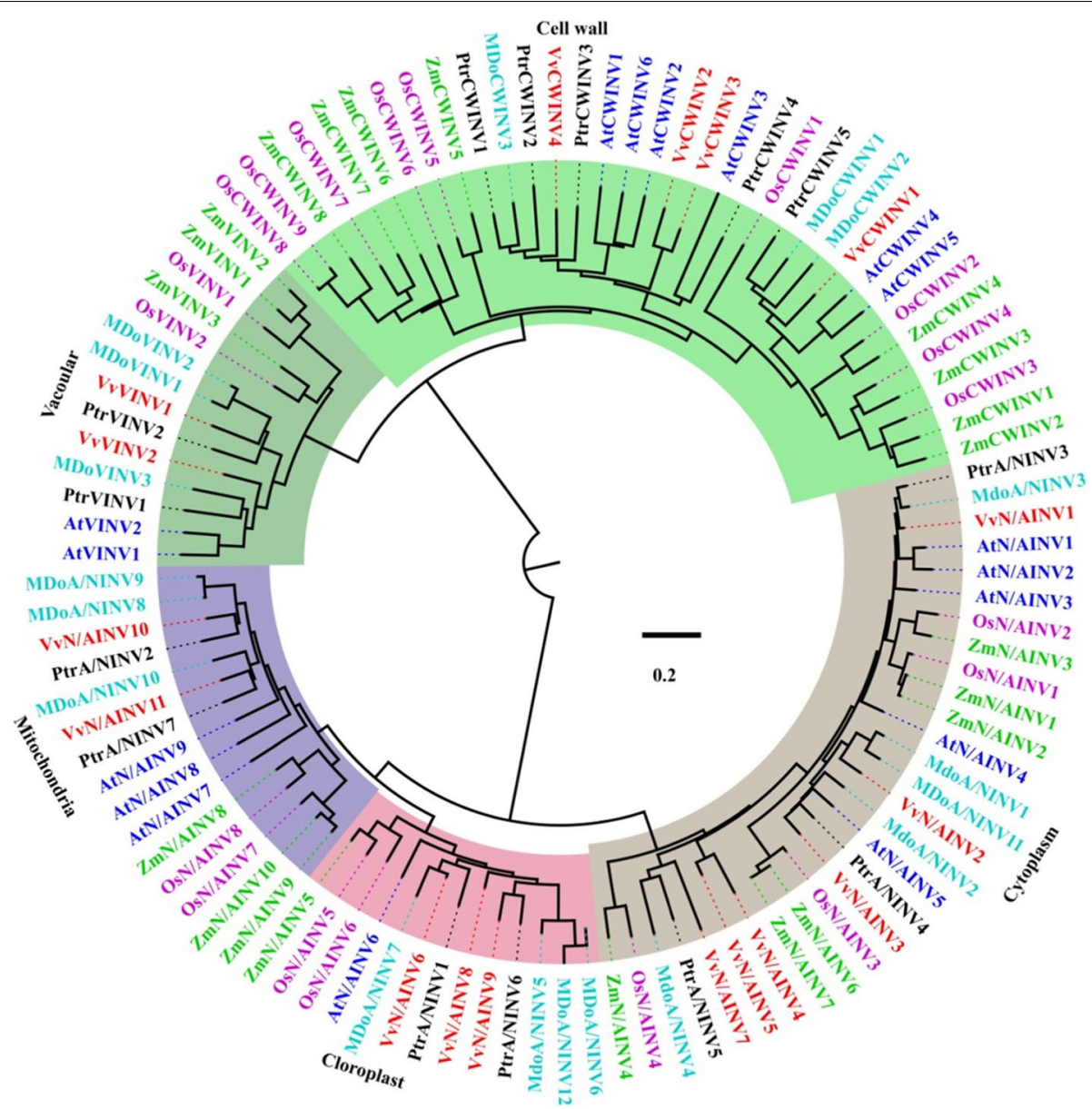

Fig. 1 Phylogenetic analysis of INV proteins between different species. Phylogenetic tree was constructed with INV proteins of Arabidopsis thaliana (blue), Oryza sativa (purple), Zea may (green), Vitis vinifera (red), Malus domestica (cyno), and P. trifoliata (black). Multiple sequences alignment of INV proteins was conducted by MEGAX with the ClustalW method. The tree was constructed with the neighbor-joining method and bootstraps with 1000 replicates for poisson correction model. The dendrogram was categorized into five distinct subfamilies that highlighted using different colors. The light brown color refers to the cytoplasm localized INV proteins. The pink and purple colored clusters represent the chloroplast and mitochondria targeted INV isoforms, respectively. The acidic INV group was divided into cell wall bound INV peptides with light green color and vacuolar INV proteins with dark green color. The scale bar indicates to the relative amount of change along the branches. Accession number can be found in Supplementary Table S2

\section{Tissue-specific gene expression analysis of PtrINV genes in P. trifoliata}

To investigate the biological role of PtrINV genes, the absolute quantification analysis was performed in different tissues according to the previous report [47]. Intriguingly, PtrINV genes were expressed in all tested tissues of $P$. trifoliata including leaves, stems, roots, fruits and flowers. In consistent with previous finding that some PtrCWINV genes were specifically expressed in sink tissues [59]. Our analysis exhibited that PtrCWINV genes were pronouncedly expressed in sink tissues (roots, fruits, and flowers) more than source tissues (leaves) (Fig. 4), suggesting the potential critical role of PtrCWINV genes in sink tissues [20]. Furthermore, PtrA/NINV genes with the exception of PtrA/NINV5 were highly expressed in leaves and stems compared to the acidic INV genes with the exception of PtrVINV1 and PtrCWINV4 (Fig. 4), indicating that PtrA/NINVs and PtrAINVs could function diversely in different tissues. Moreover, PtrA/NINV genes were also expressed highly in flowers and matured fruits (Fig. 4). Among PtrINV family members, PtrVINV2 and PtrCWINV5 had lower transcript abundance in all tested $P$. trifoliata tissues. These differential expression patterns may provide important clues for exploring their functions in different tissues in the future. 


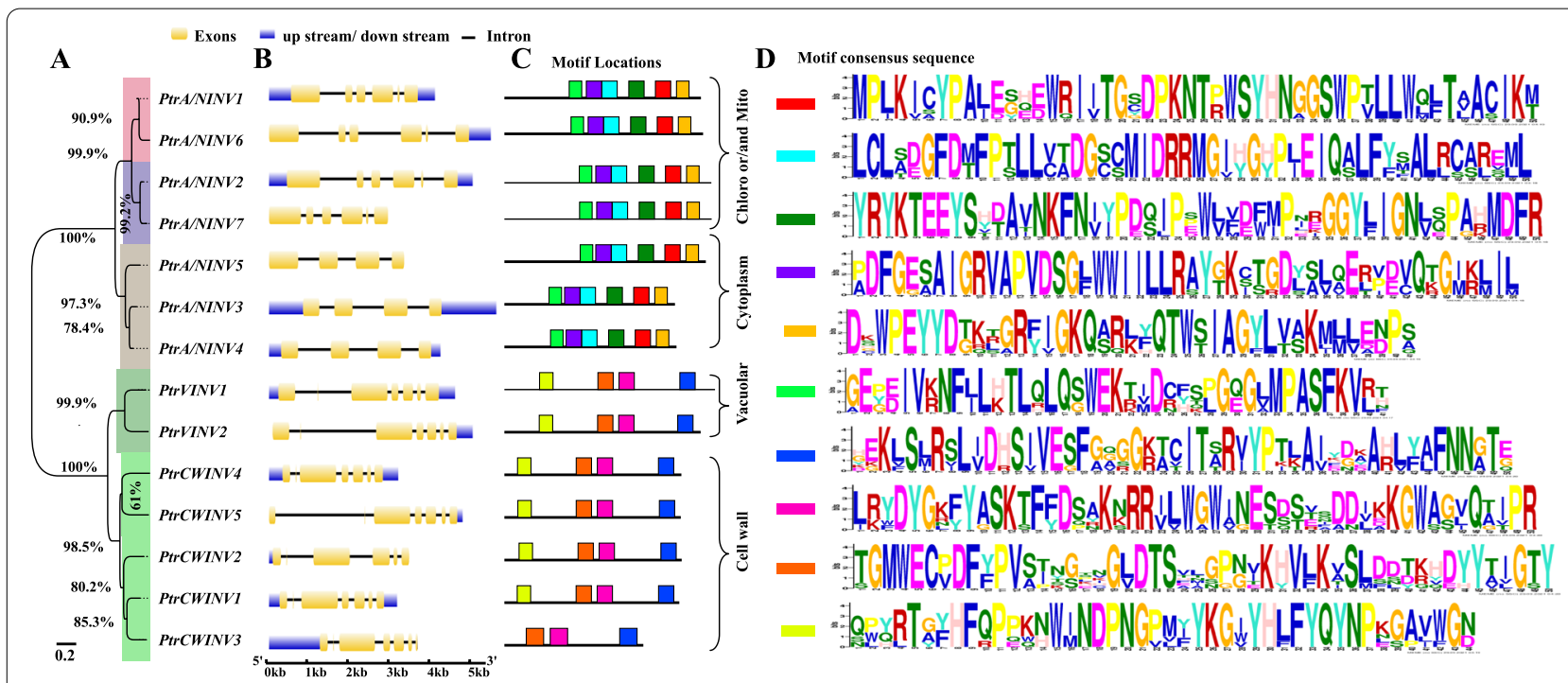

Fig. 2 Phylogenetic relationship, gene structure and protein motifs distribuation of PtrINV family members in P. trifoliata. A The phylogenetic tree was constructed by MEGAX using the neighbor-joining algorithm and bootstraps with 1000 replicates for poisson correction model. The scale bar indicates to the relative amount of change along the branches, and numbers beside the branches of dendrogram represent bootstrap values. B Gene structure was built using the Gene Structure Display Server (GSDS). Brown rectangles refer to the exons, lines indicate to the introns, and blue rectangles show the upstream and downstream untransalated regions (UTR). C The distribution of protein conserved motif. D The sequence of motif

\section{Gene expression analysis of PtrINV genes in response to osmotic stresses}

It was reported that sugars and INV activity have protective role against various stresses. To examine the contribution of PtrINV genes in response to abiotic stresses, gene expression analysis of $I N V$ family members was detected in salinity $(200 \mathrm{mM})$ and dehydration treated leaves, stems and roots. Intriguingly, gene expression pattern showed that PtrINV genes were slightly induced in salt and dehydration treated stems (Fig. $5 \mathrm{~B}$ and E). In general, PtrA/NINV genes tend to be induced in osmotictreated leaves and PtrAINV genes were high expressed in osmotic-treated roots (Fig. 5A-F). This data refers to the putative roles of PtrA/NINV genes in source organs (such leaves), and the relevance of PtrAINV genes for sugar allocation to sink tissues (roots) in response to salt and dehydration stresses. The expression profile of $I N V$ genes in salt-treated leaves showed two distinct groups where PtrA/NINV1, PtrA/NINV6, PtrVINV1, PtrCWINV2, PtrCWINV4 and PtrCWINV5 were not induced (Fig. 5A). While, the expression levels of PtrA/NINV7, PtrVINV2, PtrCWINV1 and PtrCWINV3 were highly elevated in salt-treated roots (Fig. 5C). On the other hand, the transcript abundance of PtrA/NINV1, PtrA/ NINV5, PtrCWINV2, PtrCWINV4 and PtrCWINV5 are slightly induced in dehydration-treated leaves (Fig. 5D). Furthermore, our data showed that PtrA/NINV7 and
PtrCWINV1 are constitutively expressed in response to the osmotic stresses (salt and dehydration) (Fig. 5A-F). Moreover, cis-acting regulatory elements were analyzed in the promoter sequence of INV members. As shown in Fig. S3, MYB, WRKY and MYC transcription factorsbinding sites, AT-rich motifs, light responsive-, stress responsive-, low temperature responsive- and phytohormones (such as gibberellin, abscisic acid, salicylic acid, and auxin) responsive elements were identified, suggesting that INV genes were undergone a complex transcriptional regulation in respond to various environmental conditions. Accordingly, these results revealed the potential function of PtrA/NINV7 and PtrCWINV1 in multiple abiotic stresses of $P$. trifoliata.

\section{Gene expression analysis of PtrINV genes in response to low temperature}

To test the contribution of $I N V$ genes in cold tolerance of $P$. trifoliata, we examined the gene expression of $I N V$ family members in three different tissue types (leaves, stems and roots) during cold stress $\left(4{ }^{\circ} \mathrm{C}\right.$ for $\left.72 \mathrm{~h}\right)$. In cold-treated leaves, PtrA/ NINV5, PtrCWINV2, PtrCWINV3 and PtrCWINV4 were reduced during low temperature, whereas other genes were induced under cold stress (Fig. 6A). On the other hand, the expression profile of PtrINV genes in 


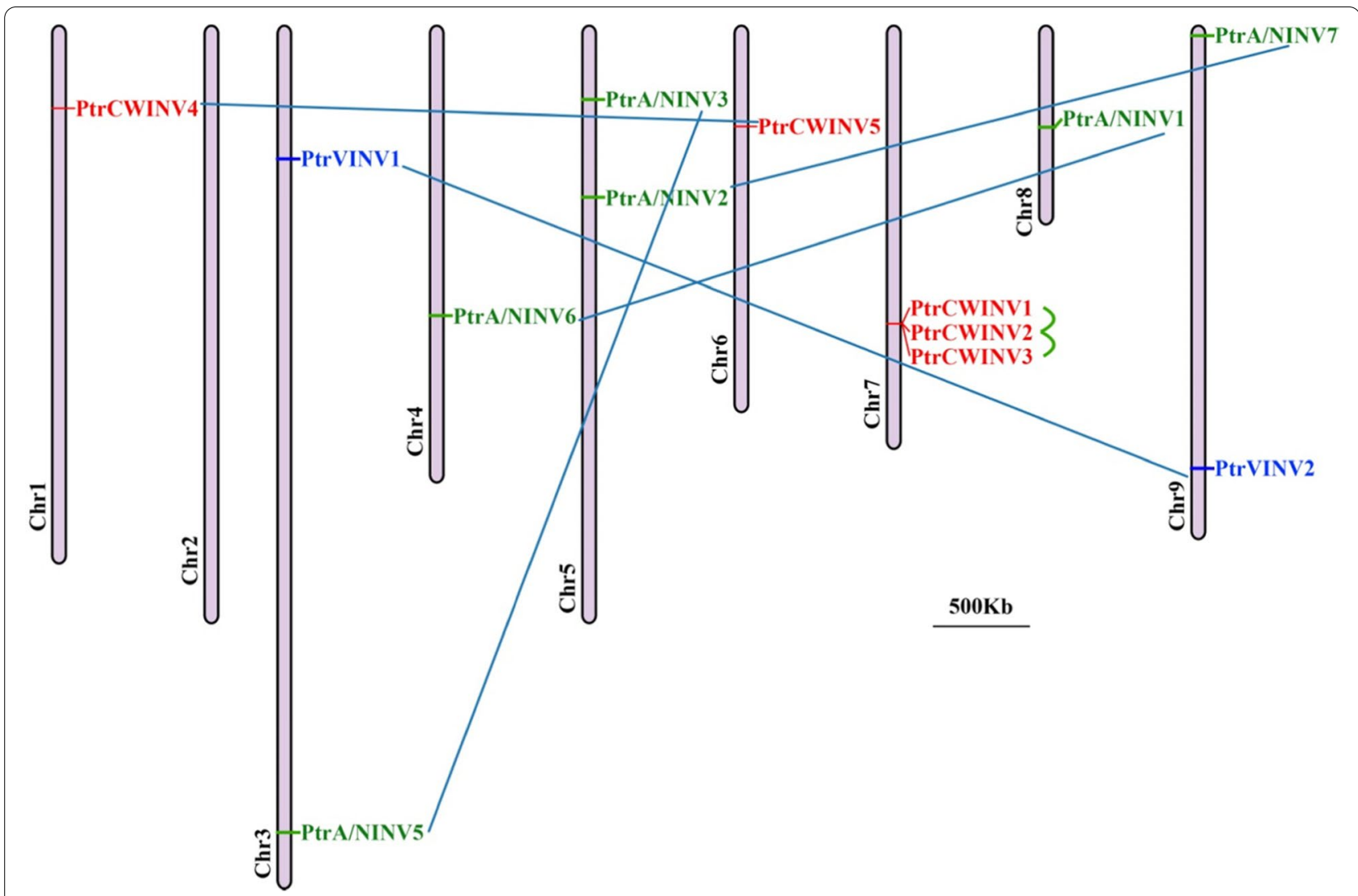

Fig. 3 Chromosomal map of PtrINV genes within the genome of $P$. trifoliata. Thirteen PtrINV genes were mapped onto eight of nine $P$. trifoliata chromosomes $(2 n=18)$. Chromosome numbers indicated below each chromosome. PtrA/NINV genes are shown in green, PtrVINV genes are in blue, and PtrCWINV genes are in red. Genome-wide or segmentally duplicated genes are linked by blue lines. Tandemly duplicated genes are linked with green lines. Scale bar is $500 \mathrm{k}$ bases $(\mathrm{kb})$

cold-treated stems was divided into highly expressed genes (including PtrA/NINV2, PtrA/NINV5, PtrA/ NINV7 and PtrCWINV1) and slightly or not induced genes (Fig. 6B). Moreover, PtrA/NINV7, PtrVINV1 and PtrCWINV1 genes among other PtrINV genes are dramatically up-regulated in cold-treated roots (Fig. 6C). In general, our analysis manifested that PtrINV genes were more induced in cold-treated leaves compared to stems and roots. Furthermore, the transcript abundance of PtrINV genes with exception of PtrA/NINV7 tends to down-regulate at the beginning of cold stress in stems and roots (Fig. 6A-C).

Intriguingly, the gene expression of PtrA/NINV7 was expressed significantly after short-term (6h) of cold stress and reach to highest level at $48 \mathrm{~h}$ in all tested tissue types compared to other PtrINV genes (Fig. 6A-C). Furthermore, the gene expression analysis of PtrCWINV genes in P. trifoliata manifested that PtrCWINV1 among other PtrCWINV genes was predominantly up-regulated at late stage of cold stress in all tested tissue types. Consequently, our data revealed the potential importance role of PtrA/NINV7 and PtrCWINV1 for cold tolerance of $P$. trifoliata.

\section{Higher sugar contents and soluble invertases activities were correlated with the freezing tolerance of Citrus species}

To obtain insight about the freezing tolerance of Citrus, we selected six Citrus and its relative species for cold tolerance assay (Ptr, P. trifoliata; Ci, Citrus ichangensis; Cs, C. sinensis; $\mathrm{Cg}, \mathrm{C}$. grandis; $\mathrm{Cl}, \mathrm{C}$. limon and $\mathrm{Fj}$, Fortunella japonica). Expectedly, P. trifoliata, C. ichangensis and C. sinensis displayed less freezing damage phenotype compared to other species, due to maintaining the integrity of photosynthetic apparatus and ROS homeostasis (Fig. S4). The dynamic changes in sugars contents were monitored after exposing two-month old seedlings to $4{ }^{\circ} \mathrm{C}$ in time-course manner $(0 \mathrm{~h}, 10 \mathrm{~h}, 24 \mathrm{~h}, 48 \mathrm{~h}$ and $72 \mathrm{~h})$. The results showed that Suc, Glc, and Frc contents were significantly accumulated in cold-tolerant species (P. trifoliata, C. ichangensis and C. sinensis) compared to that of sensitive ones (C. grandis, C. limon and $F$. japonica) after 


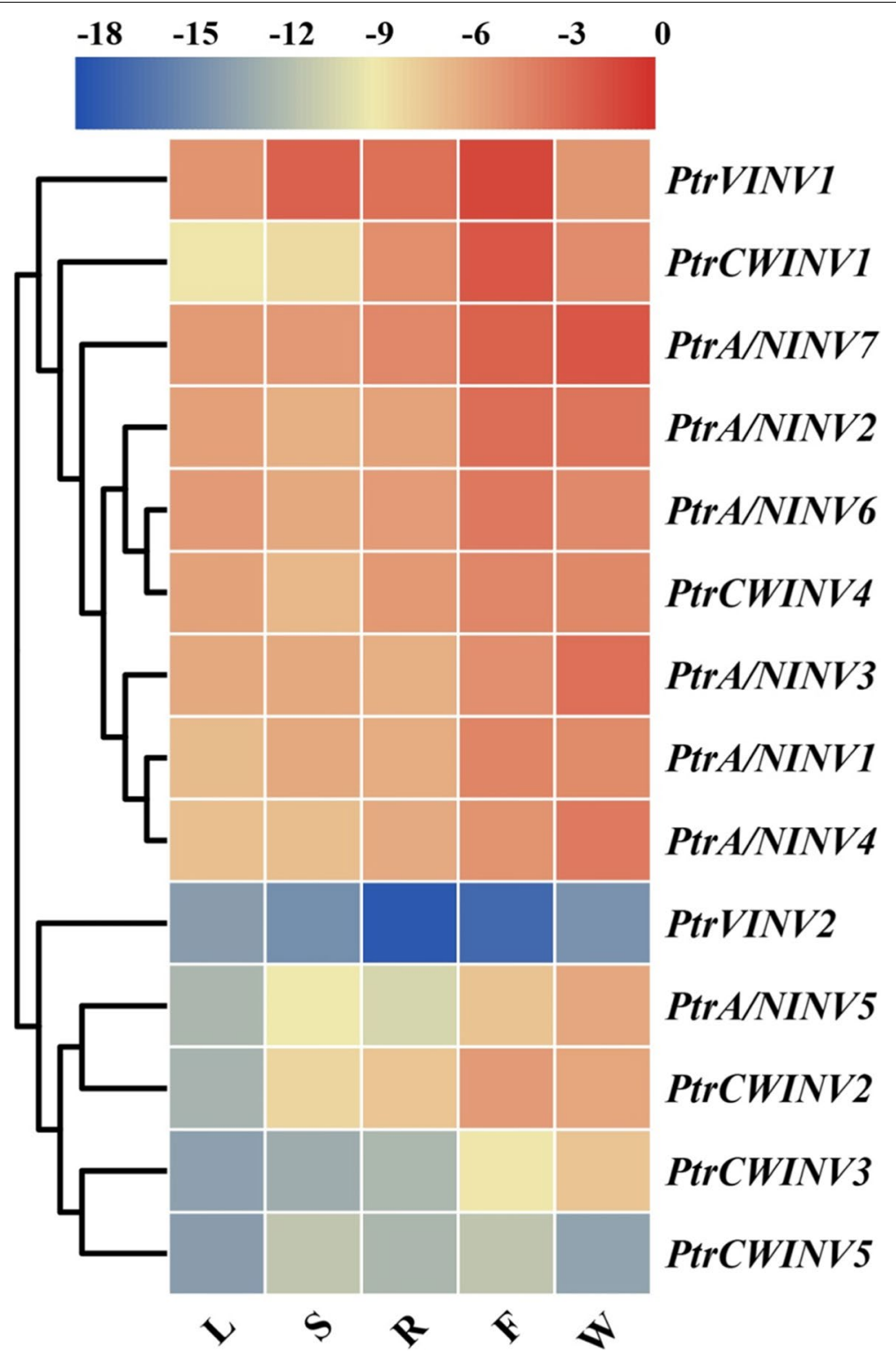

Fig. 4 Tissue-specific expression profile of PtrINV genes in P. trifoliata. RNA was extracted from two-month old leaves (L), stems (S) and roots (R) of P. trifoliata seedlings, P. trifoliata flowers (W) of spring blossom, and matured P. trifoliata fruits (F). Real-time quantitative PCR (qRT-PCR) assay was performed using specific primers and PtrActin gene was utilized as reference gene. The transcript abundance was obtained based on standard curve using QuantStudio ${ }^{\mathrm{TM}} 7$ Real-Time PCR Software v1.1 and normalization by the expression of PtrActin. Heatmap figure was constructed using $\log _{2}$ of gene expression data by TBtools based on default features: euclidean distance method, and complete cluster method $(n=3)$. The red color indicates to highly expression levels, and blue color shows low expression levels. The cluster dendrogram is shown on left side of the heatmap

cold treatment (Fig. 7A-C). Moreover, total sugar content was significantly increased in all cold-treated leaves of species, except for F. japonica, which demonstrated the high accumulation of Suc, Glc, and Frc is essential for the cold-tolerance of Citrus species.

Suc is the final byproduct of photosynthesis and it have to be hydrolyzed before its involvement in downstream pathways. INV enzyme irreversibly hydrolyzes Suc to produce Glc and Frc, while SuSy reversibly catalyzes Suc to form UDP-Glc and Frc. However, which pathway is preferable for cold tolerance is unknown. INV activities (A/NINV, VINV and CWINV) in addition to SuSy activity in the cleavage direction were investigated during cold stress. As shown in Fig. 7E-F, the soluble invertases (A/ NINV and VINV) were specifically activated in the freezing tolerant species in parallel with sugar accumulation 


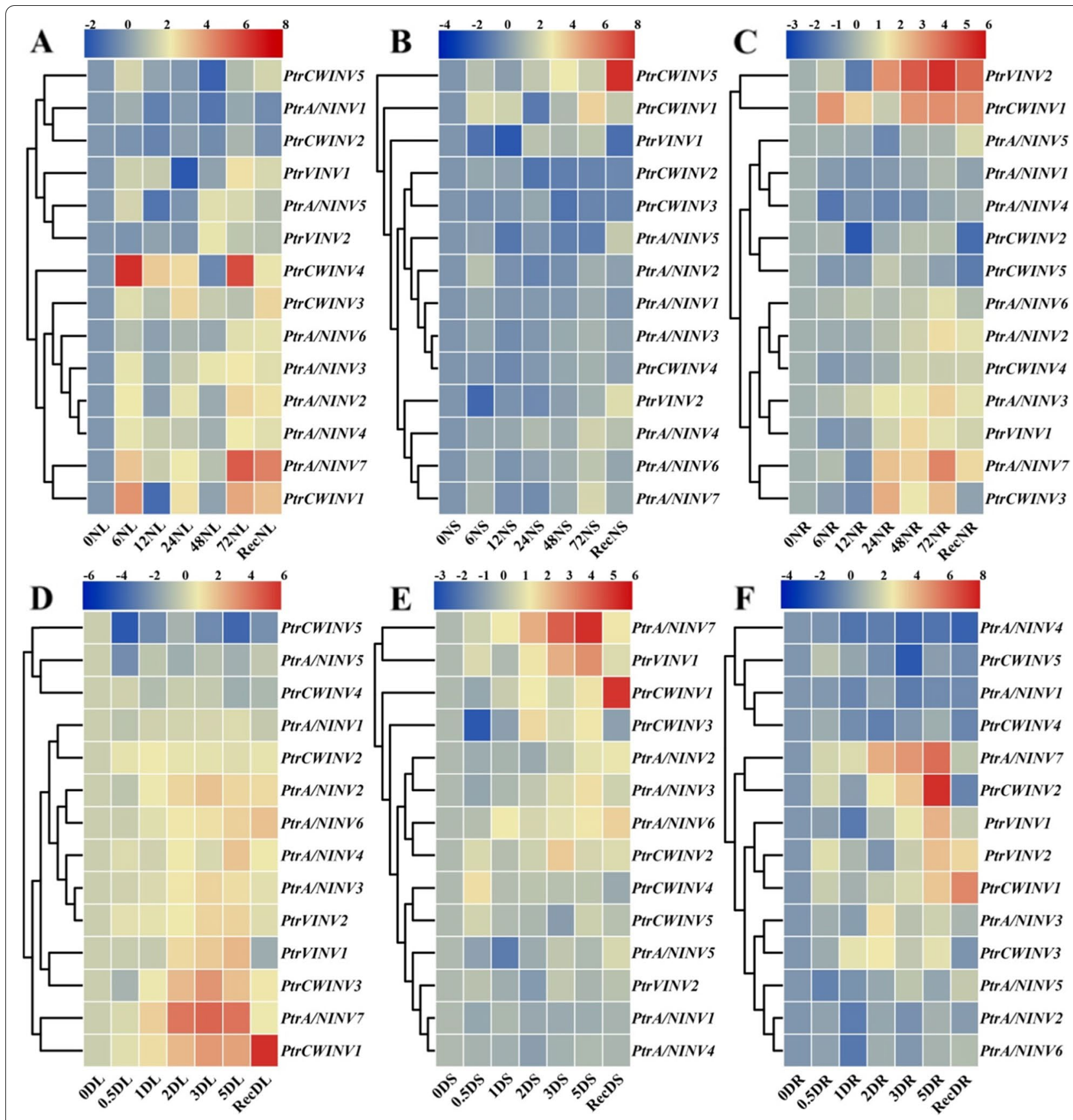

Fig. 5 The relative transcript level of PtrINV genes in different tissue types of $P$. trifoliata during osmotic stresses. A-C The transcript profile of PtrlNV genes in response to salt stress in leaves (A), stems (B) and roots (C) of two-month old P. trifoliata seedlings that exposed to $200 \mathrm{mM} \mathrm{NaCl}$ for indicated time $(0 \mathrm{~h}, 6 \mathrm{~h}, 12 \mathrm{~h}, 24 \mathrm{~h}, 48 \mathrm{~h}$ and $72 \mathrm{~h})$ and recovered for a day. (D-F) The transcript profile of PtrlNV genes in response to dehydration stress in leaves $(\mathbf{D})$, stems $(\mathbf{E})$ and roots $(\mathbf{F})$ of two-month old $P$. trifoliata seedlings that exposed to dehydration stress for indicated time $(0 \mathrm{~h}, 0.5 \mathrm{~h}$, $1 \mathrm{~h}, 2 \mathrm{~h}, 3 \mathrm{~h}$ and $5 \mathrm{~h}$ ) and recovered a day. Real-time quantitative PCR (qRT-PCR) assay was performed using specific primers and PtrActin gene was utilized as a reference gene. The gene expression analysis for each time point was repeated for at least two times with four biological replicates, and representative data are shown. The relative gene expression was calculated using the $2^{-\Delta \Delta C T}$ method relative to the initial treatment time $(0 \mathrm{~h})$ that set as 1 . The normalized values of the expression levels were converted into $\log _{2}$ of expression values and visualized by heatmap figure. Heatmap figures were constructed by TBtools based on default features, Euclidean distance method, and complete cluster method $(n=3)$ 


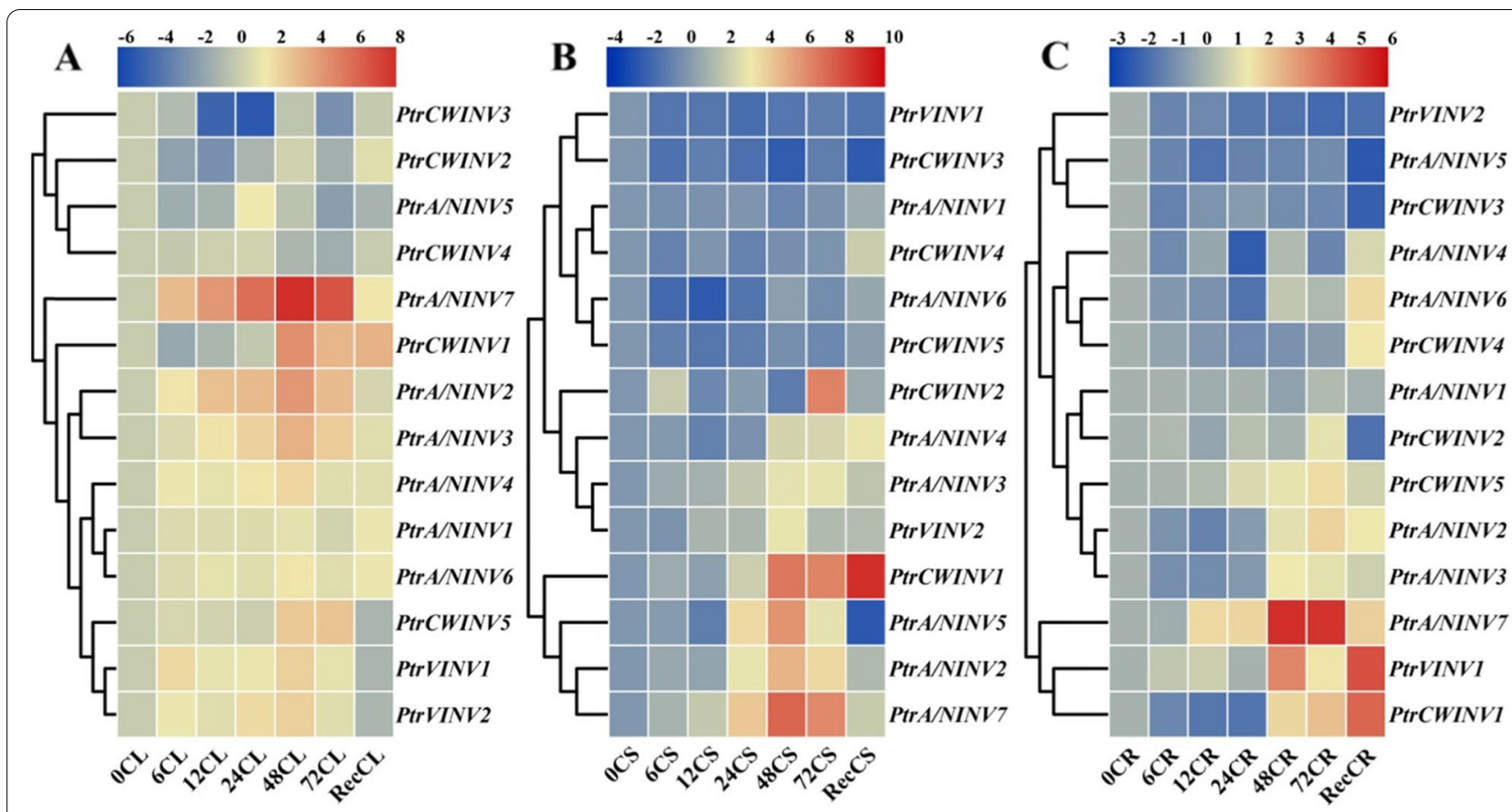

Fig. 6 The relative expression patterns of PtrINV genes in different tissue types of P. trifoliata during low temperature. RNA was extracted from $4{ }^{\circ} \mathrm{C}$ treated leaves (CL, A), stems (CS, B) and roots (CR, C) of two-month old P. trifoliata seedlings for ( $0 \mathrm{~h}, 6 \mathrm{~h}, 12 \mathrm{~h}, 24 \mathrm{~h}, 48 \mathrm{~h}, 72 \mathrm{~h})$ and recovered (Rec) at room temperature for a day. Real-time quantitative PCR (qRT-PCR) assay was performed using specific primers and PtrActin gene was utilized as a reference gene. The gene expression analysis for each time point was repeated for at least two times with four biological replicates and representative data are shown. The relative gene expression was calculated using the $2^{-\Delta \Delta C T}$ method relatively to the initial treatment time $(0 \mathrm{~h})$ that set as 1. The normalized values of the expression levels were converted into $\log _{2}$ of expression values and visualized by heatmap figure. Heatmap figures were constructed by TBtools based on default features: Euclidean distance method and complete cluster method. $(n=3)$

during low temperature, revealing that higher accumulation of Glc and Frc in tolerant species might be caused by the soluble INVs activities. Furthermore, our data showed that CWINV activity was increased in P. trifoliata, C. ichangensis, C. sinensis, C. limon and F. japonica (Fig. 7G). Intriguingly, SuSy activity was significantly reduced during cold tress in the cold-tolerant species, particularly in the most cold-tolerant species (P. trifoliata). By contrast, SuSy activity was highly induced in the sensitive species (Fig. $7 \mathrm{H}$ ), indicating that soluble INV-mediated Suc hydrolyzing is more favorable pathway for the cold tolerance of Citrus species.

It is well-known that Glc produced by INV activity undergoes phosphorylation with hexokinase (HXK) to enter glycolysis and pentose phosphate pathway, which are important pathways for ROS scavenging and energy supply [14, 31]. We hypothesized that if Glc and INV activity are positively correlated with cold tolerance, HXK activity and Glc-6- phosphate (G-6-P) content should take the same manner. To validate this notion, HXK activity and G-6-P content were also measured in the same extract crudes. In consistent with the sugar accumulation and INV activities, HXK activity and G-6-P content were noticeably elevated in the cold tolerant species compared to that of sensitive ones (Fig. 7I-J). Overall, our consequences emphasized that Suc, Glc and Frc contents were directly correlated with soluble INV activities in the cold tolerance of Citrus (Fig. 7K).

\section{The strong induction of $A / N I N V 7$ was conserved in Citrus species during cold stress}

To examine which $I N V$ isoform could correlate with the cold tolerance of Citrus, relative expression level of $I N V$ genes were determined in cold-treated seedlings of $P$. trifoliata, C. ichangensis, C. sinensis, C. grandis, $C$. limon and F. japonica. The expression profiles of $I N V$ genes under cold stress were classified into highly expressed, moderately expressed, slightly expressed, and no induction or down regulated groups (I, III, IV and II, respectively) (Fig. 8). Of note, we failed to record gene expression of A/NINV4 from C. grandis during low temperature in qRT-PCR process (Fig. 8). Interestingly, the cold-hardy species ( $P$. trifoliata) showed more induction of PtrINV genes including two cytoplasmic A/NINV genes (PtrA/NINV3 and PtrA/NINV4), two chloroplast and mitochondria localized $A / N I N V$ genes (PtrA/NINV2 and PtrA/NINV7), two VINV 


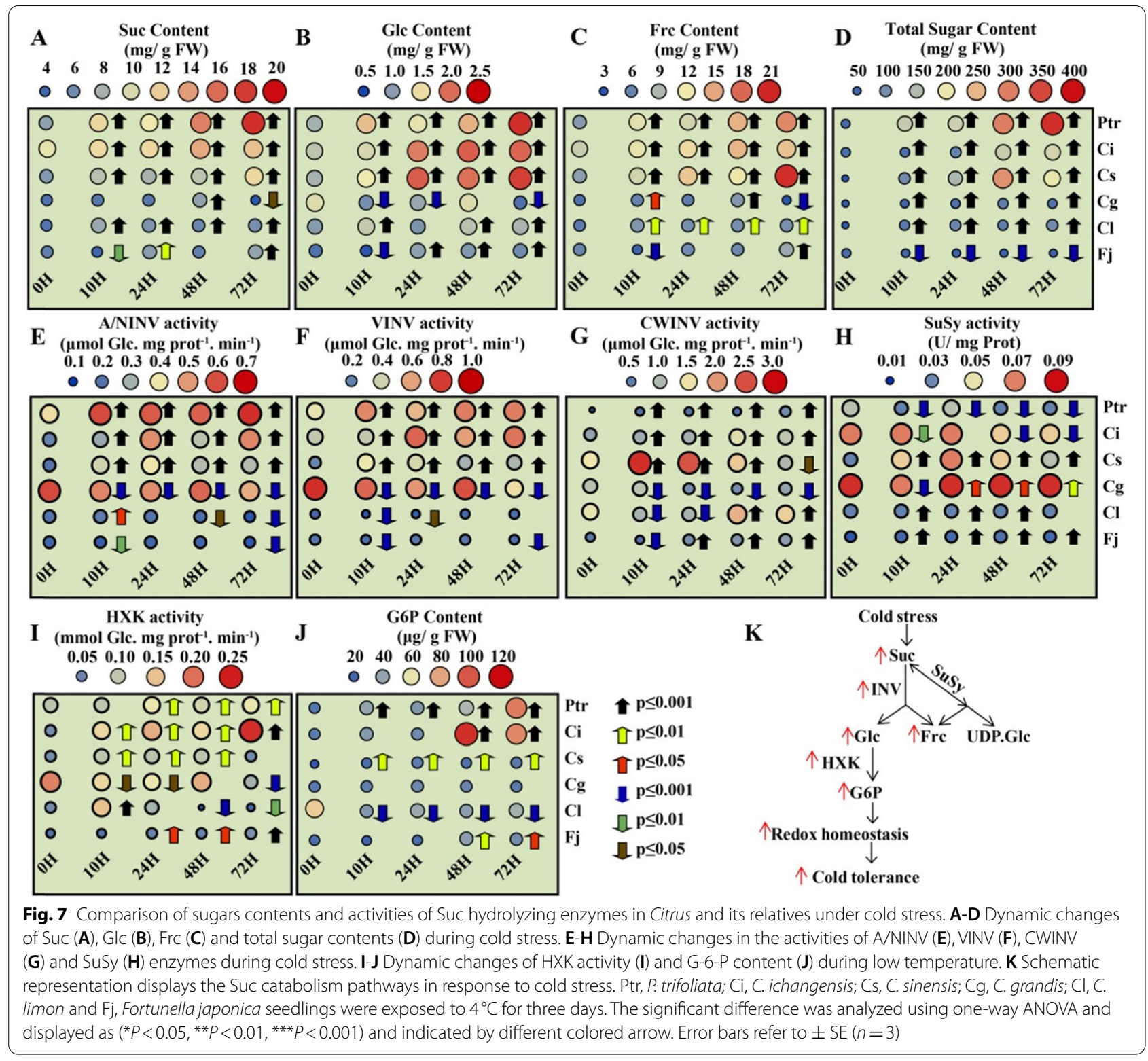

genes (PtrVINV1 and PtrVINV2) and one CWINV gene (PtrCWINV1) (Fig. 8). Contrary, the majority of INV genes were constant or repressed in the cold-sensitive F. japonica concomitant with the low accumulation of sugars during low temperatures (Figs. 7 and 8), implying to the central role of PtrINV genes in manipulating the sugar contents for cold tolerance of Citrus.

Among highly expressed genes, $A / N I N V 7$ is strongly induced after short-term low temperature treatment in the cold tolerant species (P. trifoliata, $C$. ichangensis, and C. sinensis) (Fig. 8), which accompanied with increased A/NINV enzyme activity and significant accumulation of Glc and Frc during cold stress. Overall,
$A / N I N V 7$ was implied to be a potential conserved factor for the cold tolerance of Citrus.

\section{Discussion}

In plants, invertase (INV, EC 3.2.1.26) enzymes catalyze Suc hydrolysis to produce Glc and Frc [60]. Ample evidences proofed that INVs are evolved to obtain diverse biochemical properties to fit Suc metabolism in multiple cellular compartments in response to varied conditions. Thus, INV enzymes are indispensable for plant growth, development and stress tolerance [13, 26, 31, 33, 61-63]. Various $I N V$ families were identified in previous reports, as the rice has $8 A / N I N V \mathrm{~s}, 2 V I N V \mathrm{~s}$ and $9 C W I N V \mathrm{~s}$ [16], 


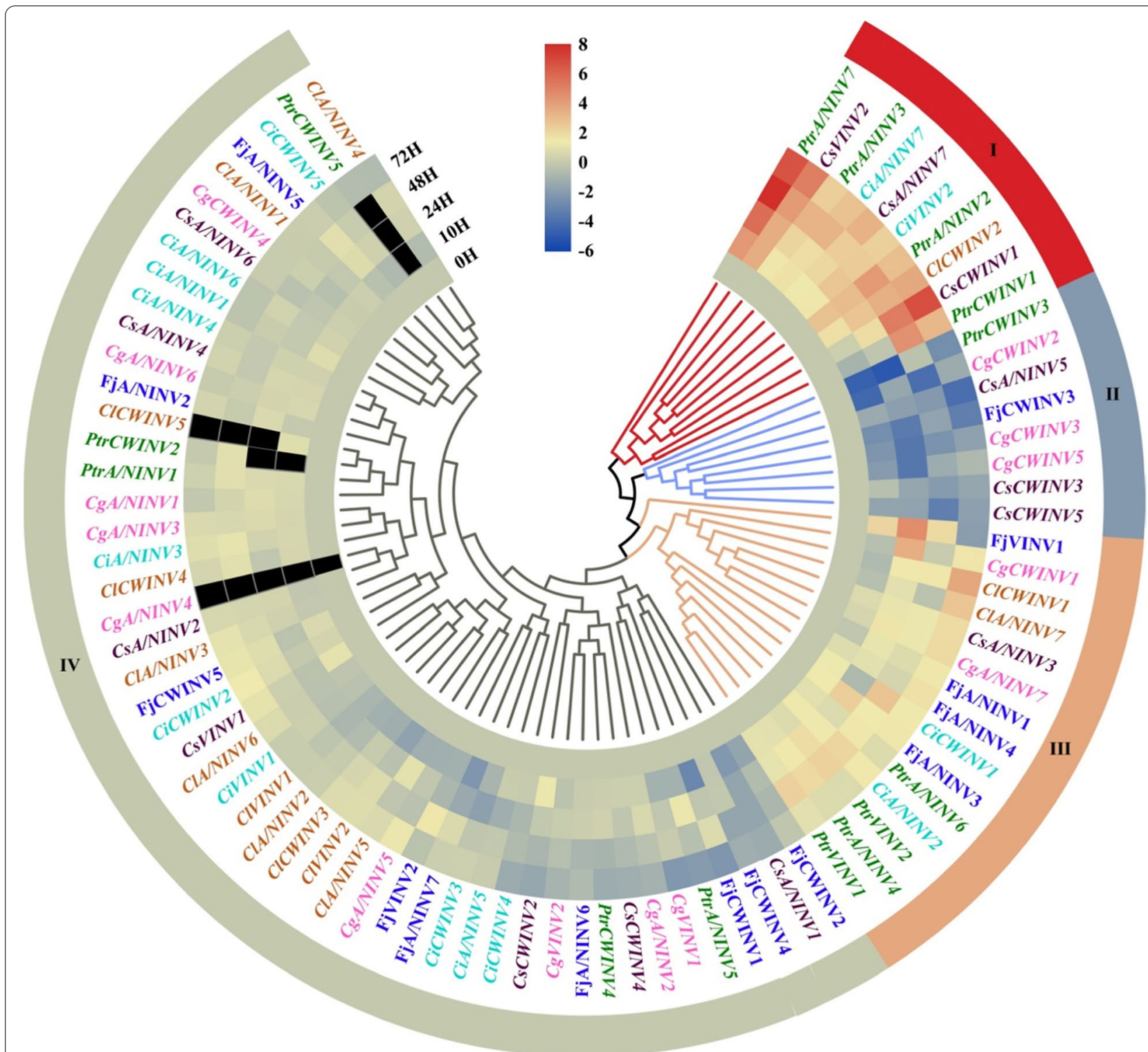

Fig. 8 Relative expression level of INV family members during low temperature in Citrus or its relative species. Two-month old seedlings from six Citrus species were treated on $4^{\circ} \mathrm{C}$ for indicated time $(0 \mathrm{~h}, 10 \mathrm{~h}, 24 \mathrm{~h}, 48 \mathrm{~h}$ and $72 \mathrm{~h})$ and the leaves were collected for further analysis. Ptr, P. trifoliata; $\mathrm{Ci}$, Citrus ichangensis; Cs, C. sinensis; Cg, C. grandis; Cl, C. limon and Fj, Fortunella japonica. Real-time quantitative (qRT-PCR) assay was performed using specific primers and PtrActin gene was utilized as a reference gene. The expression profiles of INV genes under cold stress were classified into the highly expressed genes (I group), the moderately expressed genes (III group), the slightly expressed (IV group) and no induction or down regulated genes (II group), which were colored by red, brown, gray and blue, respectively. The gene expression analysis for each treatment or each time point was repeated for at least four times. The relative gene expression was calculated using the $2^{-\Delta \Delta C T}$ method relatively to the initial treatment time $(0 \mathrm{~h})$ that set as 1 . The normalized values of the expression levels were converted into Log $_{2}$ expression values and visualized by heatmap figure. Heatmap figures were constructed by TBtools based on default features, Euclidean distance method, and complete cluster method. Dark color refers to not determined values $(n=3)$

and $A$. thaliana contains 9 A/NINVs, 2 VINVs and 6 $C W I N V$ s [59]. Furthermore, 24 putative INV genes were identified in Populus genome, including 16 A/NINVs, 3 VINVs and 5 CWINVs [64]. Additionally, tomato also has

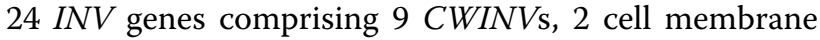
$I N V \mathrm{~s}, 11$ chloroplast $I N V \mathrm{~s}, 1$ cytosol $I N V$ and 1 vacuolar $I N V$ [21]. In fruit trees, peach genome contains 5 CWINV genes [26], and apple genome has $18 I N V$ isoforms (3 
VINVs, $3 C W I N V$ s and $12 A / N I N V s$ ) [48]. Here, the genome-wide identification of $\operatorname{PtrINV}$ gene family in $P$. trifoliata showed less diversity compared to other species, as the genome of $P$. trifoliata has 14 non-redundant $I N V$ genes including $7 \mathrm{~A} / N I N V$ genes, $2 \mathrm{VINV}$ isoforms and $5 C W I N V$ members (Table 1). This was consistent with the fact that $P$. trifoliata possesses less chromosome numbers and small genomic size $[43,45,46]$. The less abundance of PtrINV members in the P. trifoliata genome is presumably due to lower genomic duplication events that limit the expansion of the gene family.

Gene duplication ultimately culminates in producing proteins with sub-functionalization, neo-functionalization, or non-functionalization. In this report, genomewide analysis and protein sequence comparison with functionally characterized proteins were performed to estimate the potential function of PtrINV proteins. The neighbor-joining phylogenetic trees constructed for PtrINVs with Oryza sativa, Zea may, Arabidopsis thaliana, Vitis vinifera, Malus domestica INVs demonstrate their evolutionary relationships and potential similarities in function (Fig. 1). These phylogenetic trees will be a useful reference for future studies on PtrINVs. Moreover, the motif analysis and characterization will also help in future exploration of gene functions. The conservation and divergence of motif numbers present in the PtrINV proteins (Fig. 2) are expected to lead to functional similarities or differences between various PtrINV family members.

Moreover, the bioinformatics analysis illustrated that the phylogenic relationship, the introns-exon structure, and the protein motifs distribution of PtrINV genes were strongly associated (Fig. 2). Gene structure and protein motifs distribution of $P$. trifoliata $A / N I N V$ and $A I N V$ genes were conserved and similar to $I N V$ genes from other plant species $[38,64]$, revealing the significance of this gene family for plant biology [59]. The protein sequence analysis showed that PtrAINVs and PtrA/NINVs did not share any common domain due to their independent evolutionary origin [59]. Accordingly, PtrAINV and PtrA/NINV proteins were found to possess different functional domains at their C-terminus including the glyco-hydro-32 domain and the glyco-hydro-100 domain, respectively. Moreover, the $\mathrm{N}$-terminus of both subgroups are variable and have different signal peptides for several subcellular localization (Fig. S1 and Fig. S2). Furthermore, our observation exhibited that the protein sequence of PtrA/NINVs showed many subgroup-specific conserved residues in the glyco-hydro-100 domain (Fig. S1). However, the multiple sequence alignment of $P$. trifoliata AINV proteins revealed variation in the $\mathrm{C}$-terminus localized cysteine catalytic motif (MWECV/PDF) at glycohydro-32 domain (Fig. S1 and Fig. S2) [26, 38]. This catalytic motif plays essential role for Suc hydrolysis and conformation of AINV proteins [59]. Interestingly, the PtrCWINV2 protein has the MWECVDF motif similar to other vacuolar isoforms (Fig. S2), suggesting that the variation in specific residues of this conserved motif could determine the substrate specificity and/ or the optimum $\mathrm{pH}[26,64]$. However, further work is needed to characterize the function of INV proteins with consideration of the protein structure.

The important functions of INVs in plant development and stress tolerance have been widely reported. Considering that the function of any given gene is closely related to its expression, we determined the expression patterns of PtrINVs in different tissues and in response to osmotic and cold treatments to evaluate their potential functions. As expected, the comprehensive expression analysis of $I N V$ genes in $P$. trifoliata that showed the variation of expression profiles of INV isoforms in response to multiple abiotic stresses and in different tissue types (Figs. 4, 5 and 6), suggesting that each member of $I N V$ gene family could play critical role in specific tissue during specific environmental condition [59]. Interestingly, the transcript levels of $A / N I N V$ genes were more abundant in leaves compared to that of the AINV genes in P. trifoliata (Fig. 4). Furthermore, the mRNA levels of $A / N I N V$ s were highly accumulated in leaves compared to that of stems and roots in response to cold, salinity and drought stresses (Figs. 5 and 6). Consistently, higher contents of Suc, Glc and Frc were directly correlated with higher activities of soluble INV enzymes during cold stress of Citrus species (Fig. 7). Besides, leaves of the cold-tolerant Citrus species exhibited less oxidative status and sustained higher efficiency of photosynthesis (Fig. S4). It is well-known that hexoses are not transported and they accumulated in the site of formation based on the cellular demand $[12,65]$. Thus, the induction of $I N V$ genes during abiotic stresses is associated with high demand of hexoses in leaves to provide energy and osmoprotective substances. The byproducts of INV-derived Suc hydrolysis could be recruited for the protection of photosynthetic apparatus and ROS scavenging. This is more supported as several reports demonstrated that A/NINVs play more prominent role than AINVs during stressful conditions [27, 31]. Among PtrINV family members, PtrA/NINV7 responded predominantly and was up-regulated to multiple abiotic stresses in leaves, stems and roots (Figs. 5 and 6), implying its potential central role for abiotic stress tolerance. Similarly, the strong induction of $A / N I N V 7$ homologs in response to low temperature was conserved in the cold-tolerant Citrus species (Fig. 8). Thus, our insights 
indicate to the potential role of $A / N I N V$ genes for abiotic stresses tolerance in plant leaves.

\section{Conclusions}

In the present report, we performed genome-wide identification, phylogenetic analysis, and spatiotemporal expression analysis of $I N V$ genes under multiple abiotic stresses in Citrus species. Fourteen INV members were identified in P. trifoliata. Furthermore, our insights demonstrated that INVs were involved in multiple abiotic stress response and INV enzyme pathway was preferable pathway for cold tolerance of Citrus accompanying with the increase of sugars content. Overall, these results provide a framework for understanding the potential physiological roles of INV members during abiotic stresses in Citrus species.

\section{Abbreviations}

A/NINV: Alkaline/neutral invertase; AINV: Acidic invertase; VINV: Vacuolar invertase; CWINV: Cell wall invertase; Suc: Sucrose; Glc: glucose; Fru: Fructose; ROS: Reactive oxygen species.

\section{Supplementary Information}

The online version contains supplementary material available at https://doi. org/10.1186/s12870-021-03337-3.

Additional file 1: Table S1. List of primer sequences used in this study. Figure S1. Multiple sequence alignment of PtrA/NINV proteins. Figure S2. Multiple sequence alignment of PtrAINV proteins. Figure S3. Cis-acting regulatory elements analysis in the promoter of PtrINV genes. Figure S4. Freezing-tolerant Citrus species maintained the integrity of photosynthetic apparatus during freezing stress.

Additional file 2: Table S2. Accession numbers of all invertase genes from 5 plant species used in this article.

Additional file 3. The Ct values and aPCR's raw data used to construct expression profiles of PtrINV genes in P. trifoliata.

\section{Acknowledgements}

This work was supported by National Key Research and Development Program of China (2019YFD1000100), the National Natural Science Foundation of China (3172273), and Hubei Provincial Natural Science Foundation for Innovative Group (2017CFA018).

\section{Authors' contributions}

J.H.L. B.D. and C.L. designed the experiments. J.H.L. and D.G. supervised the project. B.D. achieved the experiments. B.D. W.Y. and A.A. analyzed the data and interpreted the results. B.D. wrote the manuscript. J.H.L. B.D. and C.L. revised the writing. All authors reviewed and approved the manuscript.

\section{Availability of data and materials}

The data supporting the conclusions of this article are included within the article and its additional files.

\section{Declarations}

Ethics approval and consent to participate

Not applicable.

\section{Consent for publication}

Not applicable.

\section{Competing interests}

The authors declare that they have no competing interests.

\section{Author details}

${ }^{1}$ Key Laboratory of Horticultural Plant Biology (MOE), College of Horticulture and Forestry Science, Huazhong Agricultural University, Wuhan 430070, China. ${ }^{2}$ Department of Horticulture, Faculty of Agriculture, Tishreen University, Lattakia, Syria.

Received: 6 August 2021 Accepted: 8 November 2021

Published online: 25 November 2021

\section{References}

1. Mittler R. Oxidative stress, antioxidants and stress tolerance. Trends Plant Sci. 2002;7:405-10.

2. Gechev TS, Van Breusegem F, Stone JM, Denev I, Laloi C. Reactive oxygen species as signals that modulate plant stress responses and programmed cell death. Bioessays. 2006;28:1091-101.

3. Couee I, Sulmon C, Gouesbet G, El Amrani A. Involvement of soluble sugars in reactive oxygen species balance and responses to oxidative stress in plants. J Exp Bot. 2006;57:449-59.

4. Peng T, Zhu X, Duan N, Liu JH. PtrBAM1, a $\beta$-amylase-coding gene of Poncirus trifoliata, is a CBF regulon member with function in cold tolerance by modulating soluble sugar levels. Plant Cell Environ. 2014;37:2754-67.

5. Hare PD, Cress WA, Van Staden J. Dissecting the roles of osmolyte accumulation during stress. Plant Cell Environ. 1998;21:535-53.

6. Keunen E, Peshev D, Vangronsveld J, Van Den Ende W, Cuypers A. Plant sugars are crucial players in the oxidative challenge during abiotic stress: extending the traditional concept. Plant Cell Environ. 2013;36:1242-55.

7. Ruan YL. Sucrose metabolism: gateway to diverse carbon use and sugar signaling. Annu Rev Plant Biol. 2014;65:33-67.

8. Thalmann M, Pazmino D, Seung D, Horrer D, Nigro A, Meier T, et al. Regulation of leaf starch degradation by abscisic acid is important for osmotic stress tolerance in plants. Plant Cell. 2016;28:1860-78.

9. Sicher R. Carbon partitioning and the impact of starch deficiency on the initial response of Arabidopsis to chilling temperatures. Plant Sci. 2011;181:167-76.

10. Knaupp M, Mishra KB, Nedbal L, Heyer AG. Evidence for a role of raffinose in stabilizing photosystem II during freeze-thaw cycles. Planta. 2011;234:477-86.

11. Usadel B, Blasing OE, Gibon Y, Poree F, Hohne M, Gunter M, et al. Multilevel genomic analysis of the response of transcripts, enzyme activities and metabolites in Arabidopsis rosettes to a progressive decrease of temperature in the non-freezing range. Plant Cell Environ. 2008;31:518-47.

12. Kocal N, Sonnewald U, Sonnewald S. Cell wall-bound invertase limits sucrose export and is involved in symptom development and inhibition of photosynthesis during compatible interaction between tomato and Xanthomonas campestris pv vesicatoria. Plant Physiol. 2008;148:1523-36.

13. Dahro B, Wang F, Peng T, Liu JH. PtrA/NINV, an alkaline/neutral invertase gene of Poncirus trifoliata, confers enhanced tolerance to multiple abiotic stresses by modulating ROS levels and maintaining photosynthetic efficiency. BMC Plant Biol. 2016;16:76.

14. Meng LS, Wei ZQ, Cao XY, Tong C, Lv MJ, Yu F, et al. Cytosolic invertasemediated root growth is feedback regulated by a glucose-dependent signaling loop. Plant Physiol. 2020;184:895-908.

15. Deng Y, Wang J, Zhang Z, Wu Y. Transactivation of Sus 1 and Sus 2 by Opaque2 is an essential supplement to sucrose synthase-mediated endosperm filling in maize. Plant Biotechnol J. 2020;18:1897-907.

16. Ji X, Van den Ende W, Van Laere A, Cheng S, Bennett J. Structure, evolution, and expression of the two invertase gene families of rice. J Mol Evol. 2005;60:615-34.

17. Tauzin AS, Giardina T. Sucrose and invertases, a part of the plant defense response to the biotic stresses. Front Plant Sci. 2014;5:293.

18. Vargas WA, Salerno GL. The Cinderella story of sucrose hydrolysis: alkaline/ neutral invertases, from cyanobacteria to unforeseen roles in plant cytosol and organelles. Plant Sci. 2010;178:1-8.

19. Liu YH, Offler CE, Ruan YL. Cell wall invertase promotes fruit set under heat stress by suppressing ros-independent cell death. Plant Physiol. 2016;172:163-80. 
20. Guo X, Chen H, Liu Y, Chen W, Ying Y, Han J, et al. The acid invertase gene family is involved in internode elongation in Phyllostachys heterocycla cv. Pubescens. Tree Physiol. 2020;40:1217-31.

21. Ahiakpa JK, Magdy M, Karikari B, Munir S, Mumtaz MA, Tamim SA, et al. Genome-wide identification and expression profiling of tomato invertase genes indicate their response to stress and phytohormones. J Plant Growth Regul. 2021. https://doi.org/10.1007/ s00344-021-10384-5.

22. Liu J, Han L, Huai B, Zheng P, Chang Q, Guan T, et al. Down-regulation of a wheat alkaline/neutral invertase correlates with reduced host susceptibility to wheat stripe rust caused by Puccinia striiformis. J Exp Bot. 2015:66:7325-38.

23. Jin Y, Ni DA, Ruan YL. Posttranslational elevation of cell wall invertase activity by silencing its inhibitor in tomato delays leaf senescence and increases seed weight and fruit hexose level. Plant Cell. 2009:21:2072-89.

24. Liao S, Wang L, Li J, Ruan YL. Cell wall invertase is essential for ovule development through sugar signaling rather than provision of carbon nutrients. Plant Physiol. 2020;183:1126-44.

25. Goetz M, Guivarch A, Hirsche J, Bauerfeind MA, Gonzalez MC, Hyun TK, et al. Metabolic control of tobacco pollination by sugars and invertases. Plant Physiol. 2017;173:984-97.

26. He X, Wei Y, Kou J, Xu F, Chen Z, Shao X. PpVIN2, an acid invertase gene family member, is sensitive to chilling temperature and affects sucrose metabolism in postharvest peach fruit. Plant Growth Regul. 2018:86:169-80.

27. Wang L, Zheng Y, Ding S, Zhang Q, Chen Y, Zhang J. Molecular cloning, structure, phylogeny and expression analysis of the invertase gene family in sugarcane. BMC Plant Biol. 2017;17:109.

28. Qi X, Wu Z, Li J, Mo X, Wu S, Chu J, et al. AtCYT-INV1, a neutral invertase, is involved in osmotic stress-induced inhibition on lateral root growth in Arabidopsis. Plant Mol Biol. 2007;64:575-87.

29. Vargas WA, Pontis HG, Salerno GL. Differential expression of alkaline and neutral invertases in response to environmental stresses: characterization of an alkaline isoform as a stress-response enzyme in wheat leaves. Planta. 2007;226:1535-45.

30. Martin ML, Lechner L, Zabaleta EJ, Salerno GL. A mitochondrial alkaline/neutral invertase isoform (a/N-InvC) functions in developmental energy-demanding processes in Arabidopsis. Planta. 2013;237:813-22.

31. Xiang L, Le Roy K, Bolouri-Moghaddam MR, Vanhaecke M, Lammens W, Rolland $F$, et al. Exploring the neutral invertase-oxidative stress defence connection in Arabidopsis thaliana. J Exp Bot. 2011:62:3849-62.

32. Vargas WA, Pontis HG, Salerno GL. New insights on sucrose metabolism: evidence for an active a/N-Inv in chloroplasts uncovers a novel component of the intracellular carbon trafficking. Planta. 2008;227:795-807.

33. Tamoi M, Tabuchi T, Demuratani M, Otori K, Tanabe N, Maruta T, et al. Point mutation of a plastidic invertase inhibits development of the photosynthetic apparatus and enhances nitrate assimilation in sugar-treated Arabidopsis seedlings. J Biol Chem. 2010;285:15399-407.

34. Fridman E, Zamir D. Functional divergence of a syntenic invertase gene family in tomato, potato, and Arabidopsis. Plant Physiol. 2003;131:603-9.

35. Shen LB, Qin YL, Qi ZQ, Niu Y, Liu ZJ, Liu WX, et al. Genome-wide analysis, expression profile, and characterization of the acid invertase gene family in pepper. Int J Mol Sci. 2019;20:15.

36. Yao Y, Geng M-T, Wu X-H, Liu J, Li R-M, Hu X-W, et al. Genome-wide identification, expression, and activity analysis of alkaline/neutral invertase gene family from cassava (Manihot esculenta Crantz). Plant Mol Biol Rep. 2014;33:304-15.

37. Yao Y, Geng MT, Wu XH, Liu J, Li RM, Hu XW, et al. Genome-wide identification, 3D modeling, expression and enzymatic activity analysis of cell wall invertase gene family from cassava (Manihot esculenta Crantz). Int J Mol Sci. 2014;15:7313-31.

38. Juarez-Colunga S, Lopez-Gonzalez C, Morales-Elias NC, MassangeSanchez JA, Trachsel S, Tiessen A. Genome-wide analysis of the invertase gene family from maize. Plant Mol Biol. 2018:97:385-406.
39. Biswas MK, Bagchi M, Deng X, Chai L. Genetic Resources of Citrus and Related Genera. In: al AGe, editor. The Citrus Genome, Compendium of Plant Genomes: Springer; 2020. p. 23-31.

40. Raveh E, Goldenberg L, Porat R, Carmi N, Gentile A, La Malfa S. Conventional breeding of cultivated Citrus varieties. In: Gentile A, La Malfa S, Deng Z, editors. The Citrus genome: Springer; 2020. p. 33-48.

41. Cheng FS, Roose ML. Origin and inheritance of dwarfing by the citrus rootstock Poncirus trifoliata 'flying dragon'. J Amer Soc Hort Sci. 1995:120:286-91.

42. Garcia R, Asins MJ, Forner J, Carbonell EA. Genetic analysis of apomixis in Citrus and Poncirus by molecular markers. Theor Appl Genet. 1999:99:511-8

43. Xu Q, Chen LL, Ruan X, Chen D, Zhu A, Chen C, et al. The draft genome of sweet orange (Citrus sinensis). Nat Genet. 2013;45:59-66.

44. Gong X-Q, Liu J-H. Genetic transformation and genes for resistance to abiotic and biotic stresses in Citrus and its related genera. Plant Cell Tissue Organ Cult. 2012;113:137-47.

45. Huang $Y, X u Y$, Jiang $X$, Yu H, Jia H, Tan C, et al. Genome of a citrus rootstock and global DNA demethylation caused by heterografting. Hortic Res. 2021:8:69.

46. Peng Z, Bredeson JV, Wu GA, Shu S, Rawat N, Du D, et al. A chromosomescale reference genome of trifoliate orange (Poncirus trifoliata) provides insights into disease resistance, cold tolerance and genome evolution in Citrus. Plant J. 2020;104:1215-32.

47. Peng T, Saito T, Honda C, Ban Y, Kondo S, Liu JH, et al. Screening of UV-B-induced genes from apple peels by SSH: possible involvement of MdCOP1-mediated signaling cascade genes in anthocyanin accumulation. Physiol Plant. 2013;148:432-44.

48. Hyun TK, Eom SH, Kim JS. Genomic analysis and gene structure of the two invertase families in the domesticated apple (Malus $\mathrm{x}$ domestica Borkh.). Plant Omics. 2011:4:391-9.

49. Briesemeister S, Rahnenfuhrer J, Kohlbacher O. YLoc--an interpretable web server for predicting subcellular localization. Nucleic Acids Res. 2010;38:W497-502.

50. Emanuelsson $\mathrm{O}$, Nielsen $\mathrm{H}$, von Heijne G. ChloroP, a neural network-based method for predicting chloroplast transit peptides and their cleavage sites. Protein Sci. 1999:8:978-84

51. Emanuelsson $O$, Nielsen $H$, Brunak $S$, von Heijne G. Predicting subcellular localization of proteins based on their $\mathrm{N}$-terminal amino acid sequence. J Mol Biol. 2000;300:1005-16.

52. Claros MG, Vincens P. Computational method to predict mitochondrially imported proteins and their targeting sequences. Eur J Biochem. 1996;241:779-86.

53. Small I, Peeters N, Legeai F, Lurin C. Predotar: a tool for rapidly screening proteomes for $\mathrm{N}$-terminal targeting sequences. Proteomics. 2004:4:1581-90.

54. Chen C, Chen H, Zhang Y, Thomas HR, Frank MH, He Y, et al. TBtools: an integrative toolkit developed for interactive analyses of big biological data. Mol Plant. 2020;13:1194-202.

55. Jammer A, Gasperl A, Luschin-Ebengreuth N, Heyneke E, Chu H, CanteroNavarro $E_{1}$ et al. Simple and robust determination of the activity signature of key carbohydrate metabolism enzymes for physiological phenotyping in model and crop plants.J Exp Bot. 2015:66:5531-42.

56. Tomlinson KL, McHugh S, Labbe H, Grainger JL, James LE, Pomeroy KM, et al. Evidence that the hexose-to-sucrose ratio does not control the switch to storage product accumulation in oilseeds: analysis of tobacco seed development and effects of overexpressing apoplastic invertase. J Exp Bot. 2004;55:2291-303.

57. Wang M, Dai W, Du J, Ming R, Dahro B, Liu JH. ERF109 of trifoliate orange (Poncirus trifoliata (L.) Raf.) contributes to cold tolerance by directly regulating expression of Prx 1 involved in antioxidative process. Plant Biotechnol J. 2019:17:1316-32.

58. Maddison AL, Hedley PE, Meyer RC, Aziz N, Davidson D, Machray GC. Expression of tandem invertase genes associated with sexual and vegetative growth cycles in potato. Plant Mol Biol. 1999;41:741-52.

59. Wan H, Wu L, Yang Y, Zhou G, Ruan YL. Evolution of sucrose metabolism: the dichotomy of invertases and beyond. Trends Plant Sci. 2018;23:163-77. 
60. Ruan YL, Jin Y, Yang YJ, Li GJ, Boyer JS. Sugar input, metabolism, and signaling mediated by invertase: roles in development, yield potential, and response to drought and heat. Mol Plant. 2010;3:942-55.

61. Leskow CC, Kamenetzky L, Dominguez PG, Diaz Zirpolo JA, Obata T, Costa $\mathrm{H}$, et al. Allelic differences in a vacuolar invertase affect Arabidopsis growth at early plant development. J Exp Bot. 2016;67:4091-103.

62. Wang Y, Chen J, Feng J, Qin Q, Huang J. Overexpression of a loquat (Eriobotrya japonica Lindl.) vacuolar invertase affects sucrose levels and growth. Plant Cell Tissue Organ Cult. 2015;123:99-108.

63. Xu XX, Hu Q, Yang WN, Jin Y. The roles of call wall invertase inhibitor in regulating chilling tolerance in tomato. BMC Plant Biol. 2017;17:195.
64. Xu C, Chen Z, Gao K, Su X, Rao P, An X. Genome-wide identification of the invertase gene family in Populus. PLoS One. 2015;10:e0138540.

65. Li X, Guo W, Li J, Yue P, Bu H, Jiang J, et al. Histone acetylation at the promoter for the transcription factor PuWRKY31 affects sucrose accumulation in pear fruit. Plant Physiol. 2020;182:2035-46.

\section{Publisher's Note}

Springer Nature remains neutral with regard to jurisdictional claims in published maps and institutional affiliations.
Ready to submit your research? Choose BMC and benefit from:

- fast, convenient online submission

- thorough peer review by experienced researchers in your field

- rapid publication on acceptance

- support for research data, including large and complex data types

- gold Open Access which fosters wider collaboration and increased citations

- maximum visibility for your research: over $100 \mathrm{M}$ website views per year

At BMC, research is always in progress.

Learn more biomedcentral.com/submissions 